\title{
A Probabilistic Approach for Detecting Blocking Attack in RFID Systems
}

\author{
Ehsan Vahedi, Vahid Shah-Mansouri, Vincent W.S. Wong, and Ian F. Blake \\ Department of Electrical and Computer Engineering \\ The University of British Columbia, Vancouver, Canada \\ e-mail: \{vahedi, vahids, vincentw, ifblake\}@ece.ubc.ca
}

\begin{abstract}
Radio frequency identification (RFID) is a ubiquitous wireless technology which allows objects to be identified automatically. An RFID tag is a small electronic device with an antenna and has a unique serial number. In this paper, we study the use of blocker tags by malicious attackers which can cause the nearby readers not being able to successfully receive the reply messages from the RFID tags. We mathematically model the blocker tag attack problem using information extracted from the interrogations performed by the reader. Using this analytical framework, we propose a probabilistic blocker tag detection (PBTD) algorithm to detect the presence of an attacker in the system. The probability of false alarm for the P-BTD algorithm is determined via simulation. Simulation results show that our proposed algorithm has a better performance than the thresholdbased detection algorithm in terms of using a shorter time (i.e., fewer interrogations) to detect the presence of blocker tags.
\end{abstract}

\section{INTRODUCTION}

Radio frequency identification (RFID) is a ubiquitous wireless technology which allows objects to be identified automatically. An RFID system consists of readers and objects with tags. Each tag is a small electronic device with an antenna and has a unique serial identification (ID) number. An RFID tag transmits its ID (or sometimes only portion of its ID) over the wireless channel in response to an interrogation or query message by a reader [1]. Commercial applications of RFID include: inventory checking, supply chain management, labeling products for rapid checkout at the counter, e-passport, etc. In addition, there are many other potential applications such as smart refrigerators which can recognize expired food items (e.g., milk containers with tags).

For pervasive deployment, one issue which causes the public's concern is privacy in RFID systems. Some users are concerned that when they are carrying items (e.g., clothes, medicine in a bottle, currency) embedded with RFID tags, they can be tracked by nearby readers. In that case, consumer privacy may be violated. There are various proposed schemes to address the privacy issue. One approach is to use a blocker tag [2]. When a blocker tag receives a query message from a reader, it can reply with a fake signal which prevents the reader from successfully receiving the reply messages from the neighboring tags. In this approach, tags are not removed from the items which have been sold. Instead, the customer goes to the checkout counter, pays the price and receives a bag with a blocker tag inside it. This blocker can generate and broadcast the complete (or part of the) serial numbers when being interrogated by a reader. The customer puts all the purchased items in the bag and leaves the store. On its way home, the customer cannot be tracked successfully by any readers for malicious purposes. Moreover, when the customer arrives home and brings the purchased items out of the bag, the attached RFID tags can be usable again.

However, the blocker tag approach introduces another threat to the system. A malicious attacker can launch blocking attacks in selected stores or warehouses by placing the blocker tags inside those stores. A blocker tag can generate and broadcast numerous serial numbers in order to mislead the legitimate reader and force the system to be engaged in time consuming and useless interrogation sequences. To detect this type of blocker tag attacks, an approach based on a pre-determined threshold is proposed in [2]. However, such an approach can be a time-consuming operation and it can degrade the efficiency of the system. The motivation of our work is to design an algorithm which can detect the existence of blockers in a timely manner.

The contributions of our work are as follows:

- We investigate the behavior of an RFID system in the presence of blocker tags and mathematically model the blocker tag problem using the information extracted from the interrogations performed by the reader.

- We propose a probabilistic blocker tag detection (P-BTD) algorithm to detect the presence of blocker tag in the system.

- We determine the probability of false alarm for the PBTD algorithm via simulation. Simulation results show that our proposed P-BTD algorithm has a better performance than the threshold-based detection algorithm in terms of using a shorter time (or fewer interrogations) to detect the presence of blocker tags.

The rest of the paper is organized as follows: Section II summarizes the binary tree walking algorithm and introduces two types of blocker tag attacks. Section III presents the mathematical model of the blocker tag attack problem and the proposed P-BTD algorithm. Performance evaluation and comparisons are presented in Section IV. Conclusions are given in Section V.

\section{RELATED WORK}

The blocker tag protocol and our proposed P-BTD algorithm make use of the binary tree walking algorithm. In this section, we first summarize several anti-collision protocols for RFID systems, especially the binary tree walking algorithm. We then describe the blocker tag attack. 


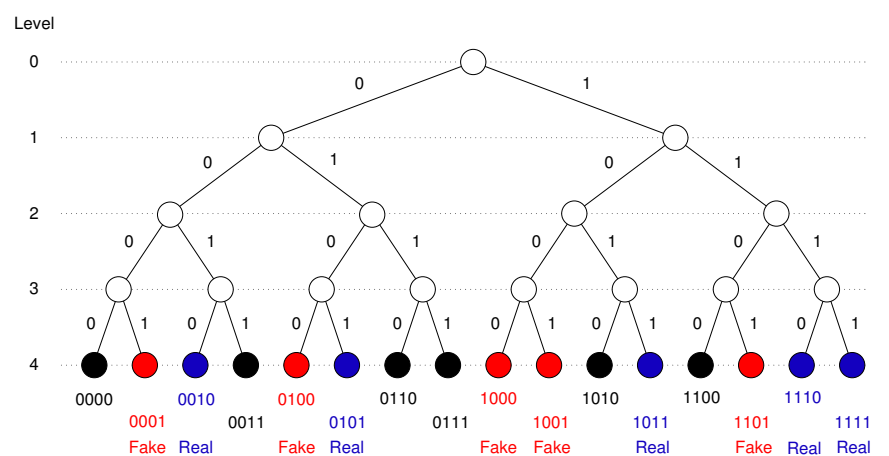

Fig. 1. Binary tree walking mechanism for tag singulation.

\section{A. RFID Anti-collision Algorithms}

RFID systems are prone to collision due to the shared nature of wireless channels. RFID anti-collision algorithms are used to improve the interrogation process. These techniques can generally be classified into tree-based algorithms and ALOHA-based algorithms. Tree-based algorithms [3][7], such as the binary tree algorithm and the query tree algorithm, continuously divide a set of tags into two subsets until each subset has only one tag. In the ALOHA-based algorithms [8]-[12], such as dynamic frame slotted ALOHA and splitting frame slotted ALOHA, a frame is divided into a number of time slots. Each tag selects a time slot and transmit with a probability. In order to reduce the probability of tag collisions, the frame size depends on the number of tags. A cardinality estimation algorithm [13] is required. Multichannel anti-collision algorithms have also been proposed for RFID readers (e.g., [14]).

\section{B. Binary Tree Walking Algorithm}

Among different tag singulation approaches, the binary tree walking algorithm has attracted a lot of attention in recent years. The blocker tag protocol in [2] is also based on the binary tree walking mechanism. Fig. 1 shows the binary tree walking mechanism with four levels (i.e., $L=4$ ). Each leaf in the tree (i.e., each node located at the $L$ th level) has a unique ID and it corresponds to the serial number of an RFID tag. The node at level 0 corresponds to the root node and it does not have an address. All other nodes located in levels 1 to $L-1$ are called the interrogation nodes.

The reader initiates the tree walking algorithm at the root of the tree. Any interrogation node at level $l$ (where $l \in$ $\{1, \ldots, L-1\}$ in this tree can be uniquely identified by a binary prefix $\boldsymbol{p}$ where

$$
\boldsymbol{p}=b_{1} b_{2} \ldots b_{l} .
$$

As an example, in Fig. 1, the interrogation nodes in level $l=2$ are identified using the prefixes of $\boldsymbol{p}=00, \boldsymbol{p}=01, \boldsymbol{p}=10$ and $\boldsymbol{p}=11$ by starting from level zero and moving along the binary tree.

Starting at a given interrogation node with prefix $\boldsymbol{p}$ at level $l$, the reader queries all tags bearing serial numbers in the leaves of the corresponding subtree; all other tags are instructed to remain silent. The queried tags reply to the reader with the $(l+1)$ st bit in their serial numbers. In other words, each tag broadcasts bit 0 if it lies in the left subtree of the interrogation node with prefix $\boldsymbol{p}$ and bit 1 if it lies in the right subtree. Consequently, if both the left and right subtrees of the interrogation node have tags present, then those of the left subtree will transmit bit 0 and those of the right subtree will transmit bit 1 . This causes a collision at the reader. In this case, the reader returns beginning at its child nodes $p \| 0$ and then $\boldsymbol{p} \| 1$, where the notation $\|$ represents concatenation. On the other hand, when all the tags reply with only a single bit $b$ (where $b \in\{0,1\}$ ), it means that they all lie on the same subtree. Therefore, the reader recurses on the node $p \| b$ and ignores the other subtrees. When the algorithm reaches a leaf, the corresponding tag transmits its $L$-bit serial ID number. The output of the binary tree walking algorithm is a list of the serial numbers of all tags within the reader's range [2].

\section{Blocker Tag Attack}

The original goal of using a blocker tag is to protect the privacy of the users when carrying purchased items (with RFID tags) on the way home [2]. However, if attackers put the blocker tags in a store or warehouse, those tags will affect the operation of the reader. There are two types of blocker tags. A universal blocker tag can simulate all possible RFID tag IDs. A partial or selective blocker tag can only simulate a selective range of IDs (e.g., the set of serial numbers assigned to a particular manufacturer). Thus, a blocker tag of this form might be used to disrupt business operations by shielding merchandise from an inventory control mechanism and delaying the whole system by forcing the reader to start a long sequence of useless interrogations.

To the best of our knowledge, the main applicable approach to detect the attack by a blocker tag is based on the use of a pre-determined threshold [2]. In this approach, the reader is expected to stall either after reaching a predefined number of interrogations, or after detecting a specific number of tags. For instance, the reader can be programmed in a way such that it stops interrogation after detecting $T+1$ tags, where $T$ can be either a large number, or the number of real (actual) RFID tags in the system. In a system without blocker tag attack, the reader should not detect more tags than the number of items equipped with RFID tags. Therefore, detecting more tags by the reader is a sign of a blocker tag attack. However, is it the best possible way of detecting the presence of an illegal blocker (attacker) in the system? This question motivated us to design a more efficient alternative in order to make the system capable of solving this problem within a shorter period of time.

\section{SYSTEM MOdEL}

The use of a pre-determined threshold to detect a blocker tag attack can be time consuming since the reader is obliged to continue searching for new serial numbers for a predefined number of interrogations. In this section, we propose a $\mathrm{P}$ BTD algorithm to detect the presence of a blocker tag. The P-BTD algorithm is a probabilistic decision making algorithm. It makes use of the information from current and previous interrogations to detect the presence of a blocker attack. The P-BTD uses the binary tree walking algorithm. 


\section{A. Probabilistic Framework}

We consider an RFID system in which there are $N$ real (actual) tags. Let $B$ denote the event that a blocker tag is present and let $\bar{B}$ denote the event that a blocker tag is not present. If a blocker tag is present, it can generate in total $F$ different fake IDs when queried by a reader. The reader uses the binary tree walking algorithm for tag singulation. The total number of levels is $L$. We use $\boldsymbol{p}=b_{1} b_{2} \ldots b_{l}$ to denote the prefix of the interrogation node. Based on the number of bits in $\boldsymbol{p}$, the corresponding level $l$ can be determined accordingly.

Let $N(\boldsymbol{p})$ and $E(\boldsymbol{p})$ denote the number of detected tags (both real and fake) and the number of empty positions in the left hand side of the interrogation node with prefix $p$, respectively. The reader maintains the values of $N(\boldsymbol{p})$ and $E(\boldsymbol{p})$, and updates those values after each interrogation. For example, when the reader reaches the interrogation node with prefix $\boldsymbol{p}=10$ in Fig. 1, it has observed $N(\boldsymbol{p})=4$ (for RFID tags with ID 0001, 0010, 0100, and 0101), and $E(\boldsymbol{p})=4$ (for empty positions $0000,0011,0110$, and 0111 ) from its previous interrogations.

When a query at interrogation node with prefix $\boldsymbol{p}$ is performed, the reader will observe one of the three possible responses. Let $r=\{0,1, c\}$ denote the response. The value of 0 and 1 corresponds to receiving bit 0 and 1 , respectively. The value of $c$ corresponds to collision. For example, at interrogation node with prefix $\boldsymbol{p}=10$ in Fig. 1, the reader observes $r=c$ as there are tags in both left and right subtrees.

Given $N(\boldsymbol{p}), E(\boldsymbol{p})$, and the presence of a blocker tag (i.e., event $B$ ), the probability of observing $r=0$ at interrogation node with prefix $\boldsymbol{p}$ is the probability that there is at least one tag in the zero branch of the subtree rooted at node with prefix $p$ and there is no tag in the one branch of this subtree. This probability can be written as

$$
\begin{gathered}
P(r=0 \mid N(\boldsymbol{p}), E(\boldsymbol{p}), B) \\
=\sum_{i=1}^{2^{L-(l+1)}}\left(\begin{array}{c}
2^{L-(l+1)} \\
i
\end{array}\right) \prod_{j=0}^{i-1}\left(\frac{[N+F-N(\boldsymbol{p})-j]^{+}}{2^{L}-N(\boldsymbol{p})-E(\boldsymbol{p})-j}\right) \\
\\
\quad \prod_{m=i}^{2^{(L-l)}-1}\left(1-\frac{[N+F-N(\boldsymbol{p})-i]^{+}}{2^{L}-N(\boldsymbol{p})-E(\boldsymbol{p})-m}\right)
\end{gathered}
$$

where $[x]^{+}=\max \{x, 0\}$. In (1), the first product term is the probability that $i$ positions in the zero branch of the interrogation node are occupied, and the second product term is the probability that the remaining $\left(2 \times\left(2^{L-(l+1)}\right)-i\right)$ positions in the zero and one branches of the interrogation node are empty.

The probability of observing $r=1$ at interrogation node with prefix $\boldsymbol{p}$ in the presence of a blocker is the probability that there is no tag in the left hand side (left branch) of the sub-tree rooted at $\boldsymbol{p}$ and there is at least one tag in the right hand side (right branch) of this sub-tree. This probability is equal to the probability of observing $r=0$. Thus,

$$
\begin{aligned}
& P(r=1 \mid N(\boldsymbol{p}), E(\boldsymbol{p}), B) \\
& \quad=P(r=0 \mid N(\boldsymbol{p}), E(\boldsymbol{p}), B) .
\end{aligned}
$$

A collision may also occur at interrogation node with prefix $p$ if there are tags in both left and right hand side of the subtree rooted at node $p$. This probability can be written as

$$
\begin{aligned}
P(r=c \mid N(\boldsymbol{p}), E(\boldsymbol{p}), B) & \\
= & 1-P(r=0 \mid N(\boldsymbol{p}), E(\boldsymbol{p}), B) \\
& -P(r=1 \mid N(\boldsymbol{p}), E(\boldsymbol{p}), B) .
\end{aligned}
$$

Given there is a blocker tag, the probability of observing $N(\boldsymbol{p})$ tags and $E(\boldsymbol{p})$ empty positions at interrogation node with prefix $\boldsymbol{p}$ is

$$
\begin{aligned}
P & N(\boldsymbol{p})=n, E(\boldsymbol{p})=e \mid B) \\
\quad & \left(\begin{array}{c}
n+e \\
n
\end{array}\right) \prod_{i=0}^{n-1}\left(\frac{N+F-i}{2^{L}-i}\right) \prod_{j=0}^{e-1}\left(1-\frac{N+F-n}{2^{L}-n-j}\right) .
\end{aligned}
$$

In (4), the first product term is the probability that $N(\boldsymbol{p})$ specified positions are occupied in the whole $(N(\boldsymbol{p})+E(\boldsymbol{p}))$ possible positions. The second product term is the probability that the remaining $E(\boldsymbol{p})$ positions are empty.

Given there is a blocker tag, the probability that the reader at interrogation node with prefix $p$ receives a response $r$, and observes $N(\boldsymbol{p})$ number of detected tags and $E(\boldsymbol{p})$ number of empty positions is

$$
\begin{aligned}
& P(r, N(\boldsymbol{p}), E(\boldsymbol{p}) \mid B) \\
& \quad=P(r \mid N(\boldsymbol{p}), E(\boldsymbol{p}), B) P(N(\boldsymbol{p}), E(\boldsymbol{p}) \mid B) .
\end{aligned}
$$

Based on the conditional probabilities in (1)-(4), the conditional probability in (5) can be determined.

Similarly, given that there is no blocker tag present in the system, we can define the probabilities $P(r \mid N(\boldsymbol{p}), E(\boldsymbol{p}), \bar{B})$, $P(N(\boldsymbol{p}), E(\boldsymbol{p}) \mid \bar{B})$, and $P(r, N(\boldsymbol{p}), E(\boldsymbol{p}) \mid \bar{B})$. We can obtain the corresponding values by substituting $F=0$ (i.e., no fake tag in the system) into (1)-(5), and replacing the event from $B$ (i.e., with blocker tag) to $\bar{B}$ (i.e., without blocker tag). Thus, we have

$$
\begin{gathered}
P(r=0 \mid N(\boldsymbol{p}), E(\boldsymbol{p}), \bar{B}) \\
=\sum_{i=1}^{2^{L-(l+1)}}\left(\begin{array}{c}
2^{L-(l+1)} \\
i
\end{array}\right) \prod_{j=0}^{i-1}\left(\frac{[N-N(\boldsymbol{p})-j]^{+}}{2^{L}-N(\boldsymbol{p})-E(\boldsymbol{p})-j}\right) \\
\quad \prod_{m=i}^{2^{(L-l)}-1}\left(1-\frac{[N-N(\boldsymbol{p})-i]^{+}}{2^{L}-N(\boldsymbol{p})-E(\boldsymbol{p})-m}\right) \\
P(r=1 \mid N(\boldsymbol{p}), E(\boldsymbol{p}), \bar{B}) \\
\quad=\quad P(r=0 \mid N(\boldsymbol{p}), E(\boldsymbol{p}), \bar{B}), \\
P(N(\boldsymbol{p})=n, E(\boldsymbol{p})=e \mid \bar{B}) \\
=\left(\begin{array}{c}
n+e \\
n
\end{array}\right) \prod_{i=0}^{n-1}\left(\frac{N-i}{2^{L}-i}\right) \prod_{j=0}^{e-1}\left(1-\frac{N-n}{2^{L}-n-j}\right)
\end{gathered}
$$

and

$$
\begin{aligned}
& P(r, N(\boldsymbol{p}), E(\boldsymbol{p}) \mid \bar{B}) \\
& \quad=P(r \mid N(\boldsymbol{p}), E(\boldsymbol{p}), \bar{B}) P(N(\boldsymbol{p}), E(\boldsymbol{p}) \mid \bar{B}) .
\end{aligned}
$$

From (6)-(8), the conditional probability in (9) can be determined. 


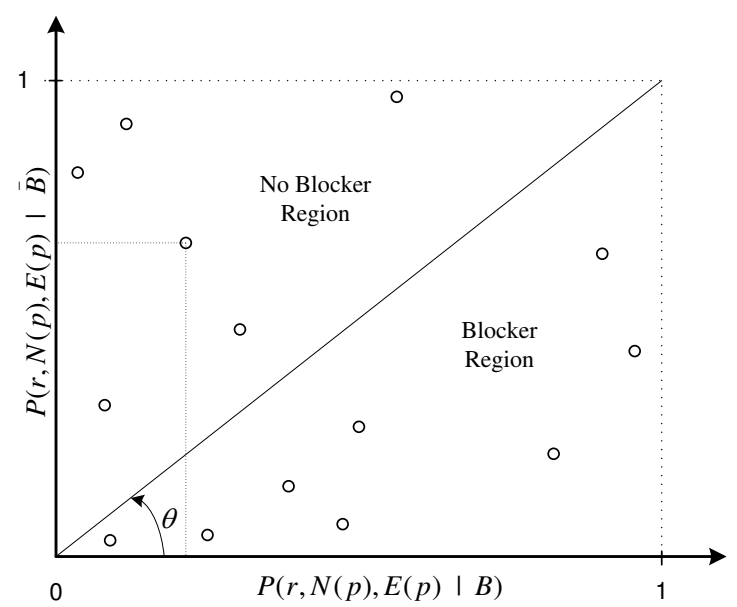

Fig. 2. Two-dimensional representation of the events and decision making criterion.

\section{B. Decision Making Criterion}

At each interrogation node with prefix $\boldsymbol{p}$, we call the tuple $(r, N(\boldsymbol{p}), E(\boldsymbol{p}))$ observed by a reader as an event. For example, in Fig. 1, the event observed at node $\boldsymbol{p}=10$ is $(r=c, N(\boldsymbol{p})=4, E(\boldsymbol{p})=4)$. Based on the observed event, the conditional probabilities in (5) and (9) can be determined.

Consider a two-dimensional space in Fig. 2, where the $\mathrm{x}$-axis and $\mathrm{y}$-axis denote $P(r, N(\boldsymbol{p}), E(\boldsymbol{p}) \quad B)$ and $P(r, N(\boldsymbol{p}), E(\boldsymbol{p}) \mid \bar{B})$, respectively. Each point (or circle) in Fig. 2 corresponds to a feasible event and its corresponding conditional probabilities in (5) and (9). Fig. 2 shows a sample of these events mapped onto this two-dimensional space. We can define two regions on the two-dimensional space. The first region is the blocker region and the second one is the no blocker region. A blocker tag (or attack) is announced if the event observed at the system resides in the blocker region in the two-dimensional space. We can distinguish between two regions by using a line with the angle of $\theta=45$ degree as depicted in Fig. 2. Thus, a reader will declare that a blocker tag exists in the system if

$$
P(r, N(\boldsymbol{p}), E(\boldsymbol{p}) \mid B)>P(r, N(\boldsymbol{p}), E(\boldsymbol{p}) \mid \bar{B}) .
$$

\section{Probabilistic Blocker Tag Detection (P-BTD) Algorithm}

Algorithm 1 shows the P-BTD algorithm to check whether there is a blocker tag (or blocker attack) in the system. The P-BTD algorithm uses binary tree walking algorithm as the singulation technique for tag detection. The values for total number of levels $L$, the threshold $T$, the step size $h$, the number of real tags $N$, and the number of fake tags $F$ are the input parameters of the algorithm. The step size $h$ is a positive integer and it denotes the number of interrogations performed between two consecutive times that the algorithm checks for the existence of a blocker tag.

The algorithm begins interrogation at the root of tree. In line 2, $N_{l}$ and $E_{l}$ are auxiliary variables used to keep track of the number of tags and empty slots detected and are used to update $N(\boldsymbol{p})$ and $E(\boldsymbol{p})$. In line 3 , the reader sends a query to all the tags in the system and waits for their responses. Based on the responses from the tags, the reader will set the response $r$ to be

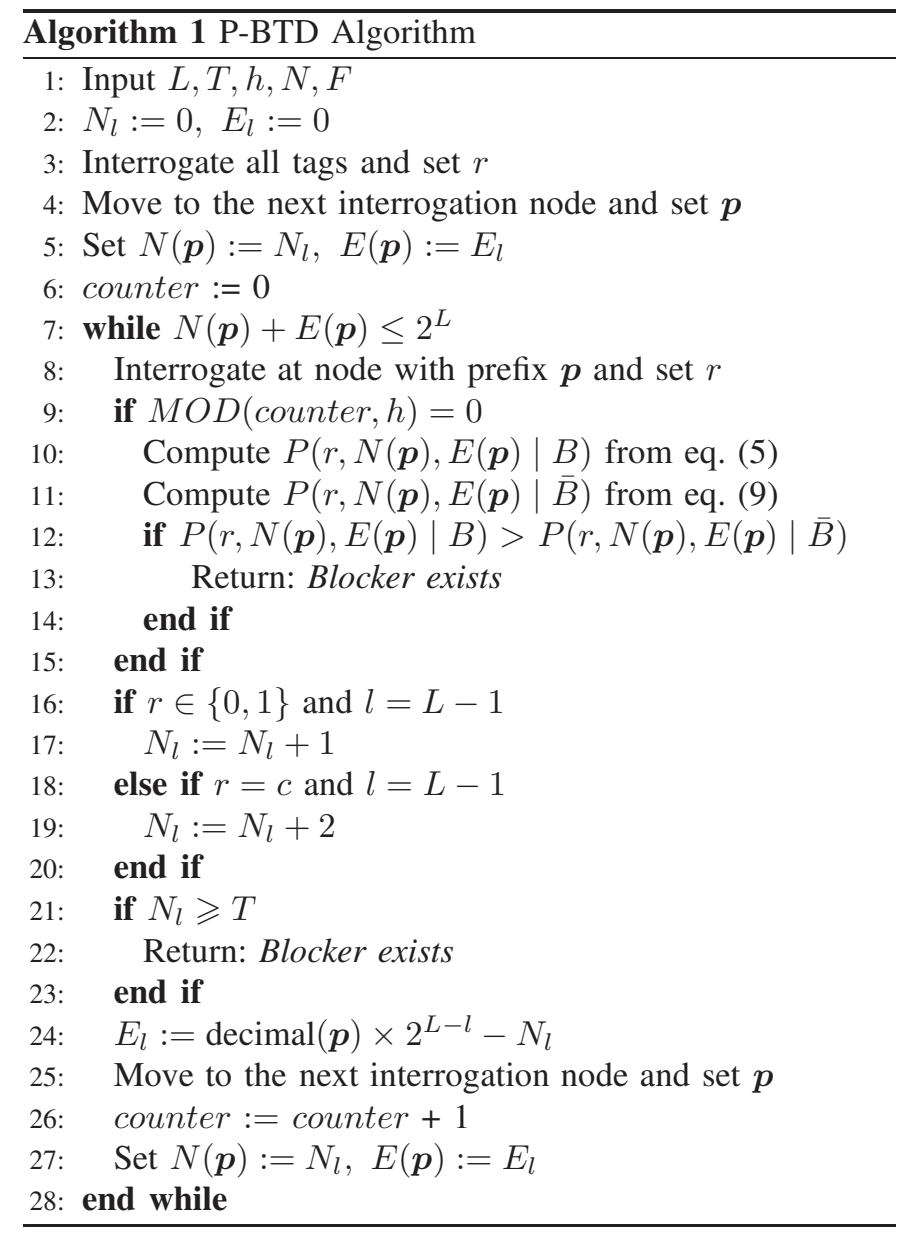

either 0,1 or $c$ (for collision). After that, the interrogation node $\boldsymbol{p}$ (i.e., the subtree) for the next interrogation is set accordingly.

In line 9, the reader checks if it needs to decide about the existence of a blocker (line 9). The modulo function $M O D$ (counter, $h)$ gives the reminder of counter divided by $h$. In lines 10 and 11 , based on the observed event, the conditional probabilities are determined from (5) and (9). In lines 12-13, if the inequality is satisfied, then the reader declares that there exists a blocker in the system and terminates the interrogation procedure.

In lines 16-20, the algorithm updates the total number of tags detected in the system. In order to do that, the algorithm checks if the interrogation node is at level $L-1$ or not. Observing a response $r$ equals to either 0 or 1 at level $L-1$ means that there exists only one tag in the two positions under that interrogation node. Therefore, $N_{l}$ is incremented by 1 . On the other hand, observing a collision at level $L-1$ means that both of the positions under the interrogation node are occupied. Therefore, $N_{l}$ is incremented by 2 .

In line 21, the algorithm also compares $N_{l}$ and $T$. If the estimation of the number of real RFID tags $N$ is accurate, then $T$ can be set to $N+1$. Otherwise, we set $T$ to a large value. In both cases, $T$ is greater than $N$ in Algorithm 1. In line 21 , if $N_{l} \geqslant T$ (i.e., the reader has detected more tags than the threshold $T$ ), then the reader declares that a blocker exists and stops interrogation. If none of the two conditions in lines 12 and 21 holds, then the reader does not have enough 


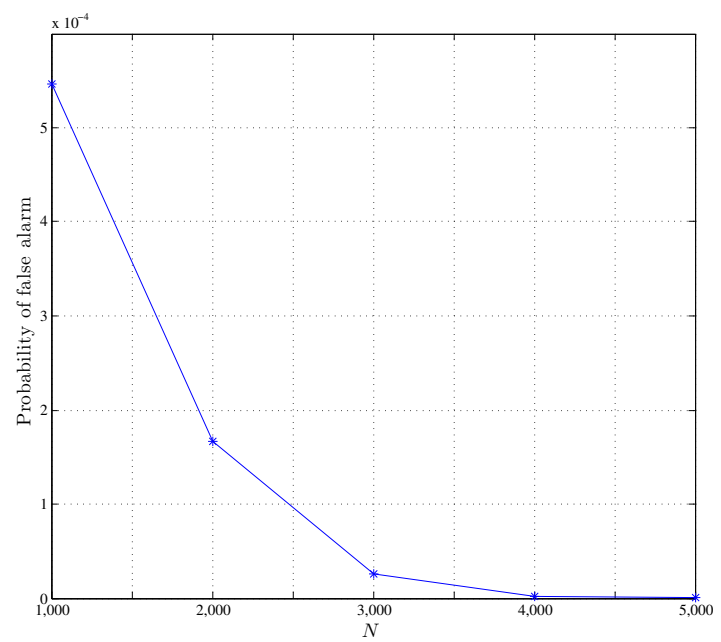

Fig. 3. Probability of false alarm by P-BTD algorithm in a 16-bit RFID system.

evidence to conclude that there exists a blocker in the system. In line 24, the algorithm calculates $E_{l}$ based on the values of $N_{l}$ and $\boldsymbol{p}$, where the function $\operatorname{decimal}(\boldsymbol{p})$ converts the binary prefix $\boldsymbol{p}$ to a decimal value. After that, the algorithm moves to the next interrogation node and goes back to line 7 . The algorithm stops either by announcing that a blocker exists or by checking all subsequent interrogation nodes in binary tree.

\section{Performance Evaluation}

In this section, we first present the simulation results to show the performance of our proposed P-BTD algorithm. We then present the performance comparison between P-BTD algorithm and the threshold-based detection algorithm [2]. All simulations are performed in MAPLE environment.

\section{A. Performance of P-BTD Algorithm}

First, we investigate the performance of the P-BTD approach in terms of probability of error. We consider an RFID system with 16-bit tag IDs (i.e, $L=16$ ). There can be two types of error in our proposed P-BTD algorithm. One is the error of missing an attacker and it happens when there exists an attacker and the algorithm cannot detect it. If the algorithm has the correct estimate of the number of real tags $N$ and the value of $T$ is set to be equal to $N+1$, then the probability of missing an attacker is zero. This is due to the fact that even if the probabilistic approach (i.e., line 12 in Algorithm 1) cannot detect an attacker, the threshold in line 21 in Algorithm 1 can detect the blocker. Therefore, the algorithm detects an attacker with probability one if it exists. The second error is the false alarm error. This can happen when the algorithm declares there is a blocker tag even though the blocker tag is not present. We calculate the probability of false alarm by simulation. In Algorithm 1, we set the input parameters as follows: $L=16, T=N+1, h=1000, F=1000$. The value of $N$ is varied from 1000 to 5000 . Fig. 3 shows the probability of false alarm. Results show that the probability of false alarm reduces when the number of real tags $N$ increases.

Next, we investigate the effect of changing parameter $F$ on the behavior of the overall system. In this experiment, the
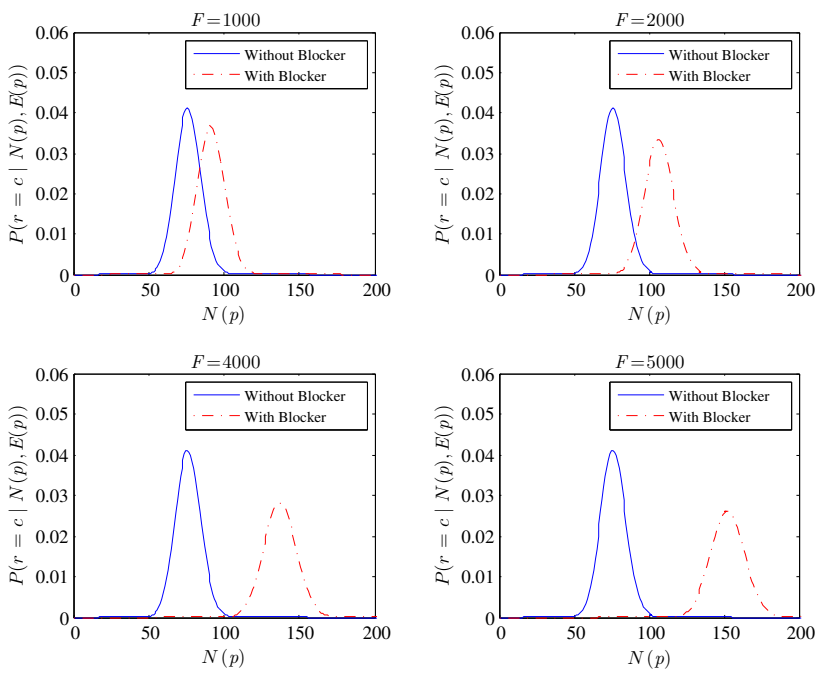

Fig. 4. The effect of changing the number of fake tags on the probability of observing collision in an RFID system with $L=16, N=5000, \boldsymbol{p}=$ 000000111110100 , and $h=1000$.

number of levels $L$ is equal to 16 , the number of real (actual) tags $N$ is 5000, and the step size $h$ is 1000 . We vary the number of fake tags $F$ between 1000 and 5000 .

Fig. 4 shows the probability of observing collision at interrogation node with prefix $\boldsymbol{p}=000000111110100$ given $N(\boldsymbol{p})$ and $E(\boldsymbol{p})$ for different values of $F$. Results from Fig. 4 show that when the number of fake tags by the attacker increases, the distance between the two peaks related to the system with and without an attacker also increases. This situation reduces the level of uncertainty in the decision and makes it easier for the algorithm to decide about the existence of an attacker in the RFID system. In other words, if the attacker reduces the number of fake tags, the two peaks in Fig. 4 merge into each other and this can increase the level of uncertainty. On the other hand, the attacker needs to block a large portion of the serial numbers in order to be able to prevent the reader from reading the real tags [2]. This makes it easier for the algorithm to detect the attacker. Although Fig. 4 is plotted for the collision event (i.e., $r=c$ ), we obtain similar results for the cases of $r=0$ and $r=1$.

\section{B. Performance Comparison}

In this section, we compare the performance of the P-BTD algorithm with the threshold-based detection algorithm [2] for an RFID system with 16-bit IDs. First, we set the number of tags $N=5000$ and the step size $h=1000$. We vary the number of fake tags $F$ from 1000 to 5000 with steps of 1000 . The performance metric is the average of the last interrogated serial number. For each algorithm, when it declares that there is a blocker tag in the system, it also saves the ID of the last detected RFID tag. For convenience of presentation, we convert the ID from binary into decimal format. The average is taken over 1000 runs. This average can be considered as an indicator of how fast the algorithm can detect the presence of a blocker tag in the system. Smaller values for the last serial number imply that the algorithm is able to detect a blocker in the system with fewer number of interrogations. On the other hand, the larger the number of interrogations, a larger 


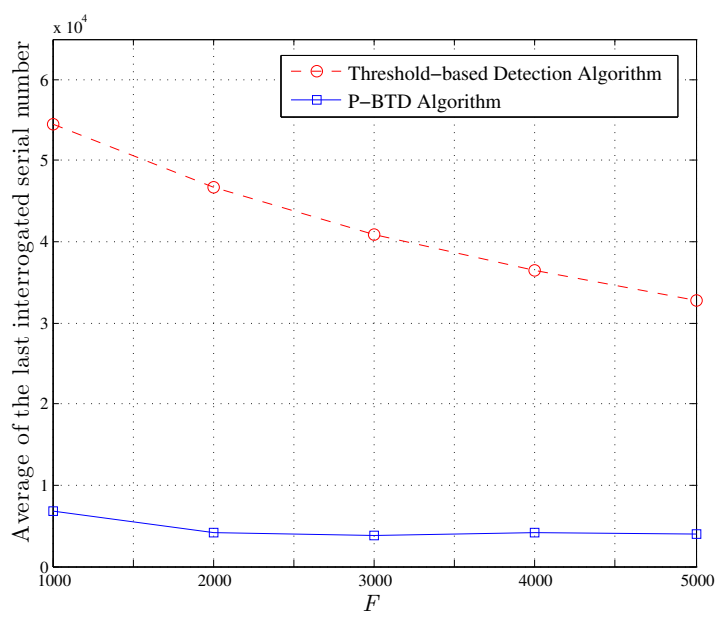

Fig. 5. Average of the last interrogated serial number before detecting the blocker versus number of fake tags $F$. ( $L=16, N=5000$ and $h=1000)$

fraction of tags (and thus larger serial numbers) it can find. In other words, the reader needs to interrogate more tags to reach to larger values of serial number. Each interrogation takes a specific amount of time to accomplish its operation. Therefore, if the reader can detect a blocker in the system with a smaller value of the last detected serial number, this means that it interrogated fewer tags (or equivalently, it spent less time) to accomplish this task.

Fig. 5 shows the results of the last interrogated serial number (in decimal format) averaged over 1000 iterations for threshold-based detection algorithm [2] and our proposed PBTD algorithm, before they detect an attacker in the system with 5000 real tags. Results show that our proposed algorithm can detect the presence of a blocker faster than the thresholdbased detection protocol [2] for different values of $F$. From Fig. 5, it can also be concluded that the detection speed of our proposed algorithm is not dependent on $F$ after values greater than 2000 . Next, we present the results by varying the number of real (actual) tags $N$. The number of fake tags $F$ is set to 5000 . The value of $N$ is varied from 1000 to 5000 with steps of 1000 . The last detected serial number (in decimal format) before the algorithm declares the presence of a blocker tag in the system is the performance metric. Results in Fig. 6 show that our proposed P-BTD algorithm is able to detect the blocker in the system with fewer iterations, resulting in a smaller average of the last interrogated serial number. As can be inferred from Fig. 6, the threshold-based detection algorithm is sensitive to the number of real tags and its detection capability is degraded by increasing the value of $N$. The detection capability of our proposed P-BTD algorithm does not depend on the the number of real tags in the system. This can add to the value of our proposed algorithm because in practical industrial setting, the algorithm should be able to work for different values of $N$ effectively.

\section{CONCLUSION}

In this paper, we modeled and formulated the blocker tag attack problem in RFID systems. We proposed a P-BTD algorithm which is a probabilistic approach for the detection of a blocker tag attack in RFID systems. At each interrogation node, the P-BTD algorithm uses information extracted from previous interrogations along with the observation at

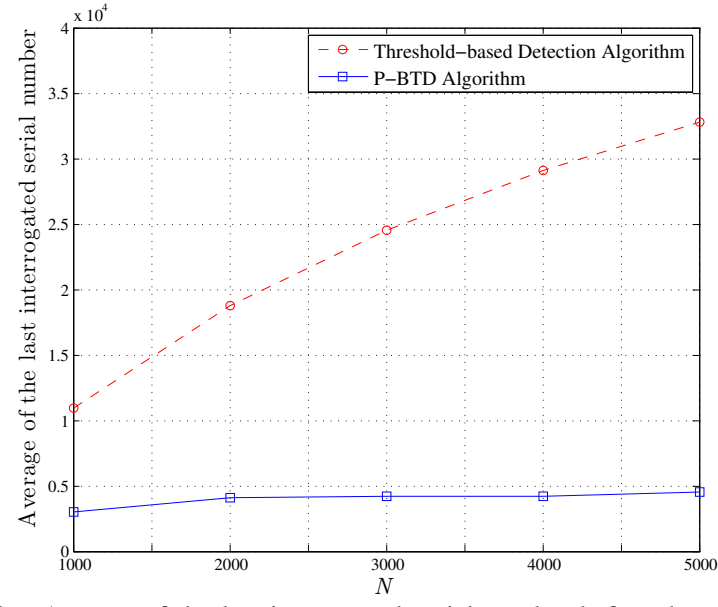

Fig. 6. Average of the last interrogated serial number before detecting the blocker versus number of real tags $N .(L=16, F=5000$ and $h=1000)$

the current interrogation node to make a decision about the existence of the blocker. We presented the simulation results of the probability of false alarm. Simulation results also showed that our proposed P-BTD algorithm can reduce the number of required interrogations for detecting a malicious blocker in the system and expedite this procedure compared to the threshold-based detection algorithm.

\section{ACKNOWLEDGEMENT}

This research is supported by the Natural Sciences and Engineering Research Council (NSERC) of Canada under grant number 364962-08.

\section{REFERENCES}

[1] S. Shepard, RFID: Radio Frequency Identification. McGraw-Hill, 2005.

[2] A. Juels, R. Rivest, and M. Szydlo, "The blocker tag: Selective blocking of RFID tags for consumer privacy," in Proc. of Computer and Communications Security, Washington, DC, Oct. 2003.

[3] Draft protocol specification for a $900 \mathrm{MHz}$ class O Radio Frequency Identification Tag. Auto-ID Center, 2003.

[4] T. P. Wang, "Enhanced binary search with cut-through operation for anti-collision in RFID systems," IEEE Communications Letter, vol. 10, pp. 236-238, Apr. 2006.

[5] J. Ryu, H. Lee, Y. Seok, T. Kwon, and Y. Choi, "A hybrid query tree protocol for RFID systems," in Proc. of IEEE ICC, China, June 2007.

[6] J. Myung and W. Lee, "Adaptive splitting protocols for RFID tag collision arbitration," in Proc. of ACM MobiHoc, Italy, May 2006.

[7] J. S. Choi, H. Lee, D. W. Engels, and R. Elmasri, "Robust and dynamic bin slotted anti-collision algorithms in RFID systems," in Proc. of IEEE Int'l Conf. on RFID, Las Vegas, NV, Apr. 2008.

[8] Y. Lai and C. Lin, "Two blocking algorithms on adaptive binary splitting: single and pair resolutions for RFID tag identification," IEEE/ACM Trans. on Networking, vol. 17, pp. 962-975, June 2009.

[9] H. Vogt, "Efficient object identification with passive RFID tags," Lecture Notes in Computer Science, vol. 2414, Jan. 2002.

[10] J. Park, M. Y. Chung, and T. J. Lee, "Identification of RFID tags in framed-slotted ALOHA with robust estimation and binary selection," IEEE Communications Letter, vol. 11, pp. 452-454, May 2007.

[11] C. Floerkemeier, "Bayesian transmission strategy for framed Aloha based RFID protocols," in Proc. of IEEE Int'l Conf. on RFID, Grapevine, TX, Mar. 2007.

[12] J.-B. Eom, T.-J. Lee, R. Rietman, and A. Yener, "Efficient framed-slotted Aloha algorithm with pilot frame and binary selection for anti-collision of RFID tags," IEEE Commun. Letters, vol. 12, pp. 861-863, Nov. 2008.

[13] V. Shah-Mansouri and V.W.S. Wong, "Anonymous cardinality estimation in RFID systems with multiple readers," in Proc. of IEEE Globecom, Honolulu, HI, Dec. 2009.

[14] A. H. Mohsenian-Rad, V. Shah-Mansouri, V.W.S. Wong, and R. Schober, "Distributed channel selection and randomized interrogation algorithms for large-scale and dense RFID systems," IEEE Trans. on Wireless Communications, 2010. 